\title{
Índice ganglionar y número de linfonodos metastásicos como factores pronósticos en cáncer de colon*
}

\author{
Drs. GUILLERMO BANNURA C. ${ }^{1}$, CLAUDIO VARGAS S. ${ }^{1}$, ALEJANDRO BARRERA E. ${ }^{1}$, \\ CARLOS MELO L. ${ }^{1}$, JAIME CONTRERAS P. ${ }^{1}$ \\ 1 Servicio y Departamento de Cirugía, Hospital Clínico San Borja Arriarán. Campus Centro, Facultad de Medicina, \\ Universidad de Chile. \\ Santiago, Chile.
}

\begin{abstract}
\section{Lymph node index as a prognostic factor for survival in stage III colon cancer}

Background: The lymph node ratio in malignant tumors corresponds to the ratio between the number of involved lymph nodes and the number of examined lymph nodes. This ratio may be a good prognostic index in stage III colon cancer. Aim: To compare the lymph node ratio with the absolute number of positive lymph nodes as prognostic factors in stage III colon cancer. Material and Methods: Analysis of 115 patients aged 25 to 91 years (63\% women) with a stage III colon cancer operated between 1991 and 2007. Survival according to the absolute number of positive lymph nodes and the lymph node index was calculated. The area under receiver operating characteristic (ROC) curves obtained after a COX regression analysis of survival, was used to analyze the prognostic value of each parameter. Results: Lymph node involvement was classified as T2 in three, T3 in 93 and T4 in 19 patients. The mean number of positive lymph nodes was 3.4 (range 1 to 34) and the mean lymph node index was $0.237 \pm 0.197$ (range $0.031-0.882$ ). Seventy four percent of patients had one to three positive lymph nodes and $24 \%$ had more than three. During a mean follow up of 67 months (range 5-216), 29 patients died. In survival analysis, the area under the ROC curve for the number of involved lymph nodes $(0.703,95$ confidence intervals (CI) $0.58-0.82)$ was slightly better than the area for lymph node index $(0.69,95 \%$ CI $0.57-0.81)$. Using a dichotomy analysis, a lymph node index over 0.31 had a higher discriminating value for survival (odds ratio (OR) 19.96 91\% CI 1.51-253.6) than the presence of 12 or more involved lymph nodes (OR $2.5595 \%$ CI 0.86-7.55). Conclusions: The lymph node index and the absolute number of involved lymph nodes are prognostic factors in stage III colon cancer
\end{abstract}

Key words: Lymph node index, colon cancer, lymph nodes.

\section{Resumen}

Introducción: El índice ganglionar (IG) se ha propuesto como un factor pronóstico mejor que el número de LN positivos en cáncer de colon estadio III. El objetivo es comparar estos factores en una serie clínica. Pacientes y Método: Se incluyen todos los pacientes estadio III resecados con intención curativa (R0). Se compara la sobrevida según el número de LN positivos y el IG mediante el análisis de las curvas ROC.

*Recibido el 27 de diciembre de 2010 y aceptado para publicación el 4 de abril de 2011.

Correspondencia: Dr. Guillermo Bannura C.

Las Limas 1622, Santiago, Chile.

gbannura@vtr.net 
Resultados: Se trata de 115 pacientes con un promedio de edad de 67,9 años (extremos 25-91), el 63,4\% mujeres. El compromiso en profundidad del tumor fue T2 en 3 casos, T3 en 93 casos y T4 en 19. El promedio de ganglios positivos fue 3,4 (extremos 1-34). El índice ganglionar promedio fue 0,237 (DE: 0,197; extremos $0,031-0,882$ ) y la mediana fue 0,1666 . El $74 \%$ de los pacientes tenía 1 a 3 ganglios positivos (N1) y el 26\% 4 o más ganglios positivos (N2). El seguimiento promedio fue de 67 meses (extremos 5-216), durante el cual fallecen 29 pacientes. El área bajo la curva ROC del número de LN afectados (0,703; IC 95\%:0,58-082) fue levemente mayor que el área bajo la curva ROC del IG (0,690; IC 95\%:0,57-0,81) (p=0,63). Al compararlas en forma dicotómica, el IG (OR: 19,96; IC 95\%:1,51-253,6) muestra una mayor capacidad de discriminación que el número de LN afectados (OR: 2,55; IC 95\%: 0,86-7,55). Conclusión: El número de LN metastásicos y el IG son factores pronósticos relevantes en la planificación de la adyuvancia del cáncer de colon estadio III.

Palabras clave: Cáncer de colon, factores pronósticos, índice ganglionar.

\section{Introducción}

El compromiso metastásico de los linfonodos se considera el factor pronóstico de mayor relevancia en el cáncer de colon (CC) sometido a resección radical con intención curativa, es decir, en ausencia de metástasis a distancia ${ }^{1,2}$. Los pacientes con LN positivos (estadio III) constituyen una categoría muy heterogénea, motivo por el cual se han segregado según el número de LN afectados. Este factor estratifica los pacientes del estadio III en grupos de diferente pronóstico, lo que ha llevado a sucesivas modificaciones del sistema de estadificación vigente. Mientras el TNM 2002 separa los pacientes en 2 grupos (1 a $3=\mathrm{N} 1$, 4 o más $\mathrm{LN}$ positivos $=\mathrm{N} 2)$, la nueva propuesta TNM 2010 reconoce 4 grupos pronósticos en el estadio III (1, 2 a 3, 4 a 6 y 7 o más LN afectados $)^{3,4}$. Sin embargo, esta categorización no considera el número de linfonodos examinados, factor que ha mostrado en algunos estudios ser un factor pronóstico independiente ${ }^{1,5,6}$. Considerando que el número de LN incluidos en la pieza operatoria es variable, algunos autores han propuesto el índice ganglionar $(\mathrm{IG}=$ número de $\mathrm{LN}$ positivos dividido por el número total de LN examinados) como un factor pronóstico independiente y de mayor potencia que el número de $\mathrm{LN}$ afectados ${ }^{7-14}$, lo que ha sido confirmado en una revisión sistemática reciente ${ }^{15}$.

El objetivo de este estudio es comparar el número de LN positivos y el IG como factores pronósticos en un grupo no seleccionado de pacientes intervenidos por un cáncer de colon en estadio III con intención curativa.

\section{Pacientes y Método}

Se incluyen todos los pacientes portadores de un adenocarcinoma de colon estadio III intervenidos con intención curativa (R0) en forma consecutiva en el período 1991-2007. Todos los pacientes fueron sometidos a una resección estándar con linfa- denectomía regional de acuerdo a la localización del tumor. Se excluyen los pacientes con un cáncer de recto y los tumores originados en una poliposis. El estudio de la pieza operatoria fue realizado de acuerdo a las normas de la Institución, incluyendo un corte por cada linfonodo. Para la etapificación clínico-patológica se empleó la $6^{\mathrm{a}}$ edición de la clasificación de la AJCC/UICC, conocida como TNM $2002^{3}$, que distingue los pacientes en estadio III con compromiso de 1 a 3 linfonodos positivos (N1) de aquellos con 4 o más ganglios afectados (N2).

El grado de diferenciación tumoral se categorizó en bien o moderadamente diferenciado y poco diferenciados e indiferenciados. La concentración sérica del CEA fue determinada por la técnica de inmunoenzimoanálisis de micropartículas (MEIA, Abbott Laboratories, Chicago, Il), aceptando el valor de $5 \mathrm{ng} / \mathrm{ml}$ como valor normal máximo. EL CEA preoperatorio se analizó como variable continua y categórica. El tamaño tumoral se midió en la pieza fijada y estudiada como variable categórica (igual o menor de $5 \mathrm{~cm} /$ mayor de $5 \mathrm{~cm}$ ). La quimioterapia adyuvante se estandarizó desde el año 1997 con 6 ciclos de 5-FU más leucovorina ${ }^{16}$, agregando FOLFOX en pacientes estadios IIIC (4 o más LN positivos) a partir de 2007. El seguimiento se efectuó en forma selectiva, incluyendo examen clínico, colonoscopia, CEA postoperatorio y TAC de abdomen y pelvis en forma no sistemática.

\section{Análisis estadístico}

Las variables continuas fueron comparadas mediante el test de Wilcoxon y las variables categóricas mediante el test de $\chi^{2}$. Las curvas de sobrevida fueron estimadas según el método de Kaplan-Meier. Para comparar las curvas de sobrevida se empleó el test log-rank. Para determinar los factores pronósticos más relevantes en un análisis multivariado se utilizó el modelo de regresión de Cox. Tanto el número de LN positivos como el IG fueron estudiados como variables continuas comparadas mediante una curva ROC para determinar el modelo con la mayor potencia pronóstica. Se estimó también la estadística 
$\mathrm{C}$ de Harrell ${ }^{17}$, que es el equivalente a la estadística $\mathrm{C}$ de concordancia correspondiente al área bajo la curva estimada en las curvas ROC para modelos de sobrevida. Para la construcción de los intervalos de confianza de esta estadística se usó bootstrap ${ }^{18}$. Como los resultados son similares sólo se muestran las curvas ROC.

\section{Resultados}

Las características clínico-patológicas de 115 pacientes intervenidos por un CC estadio III en forma consecutiva se muestran en la Tabla 1. El promedio de edad de la serie fue 67,9 años (extremos 25-91). No hubo diferencias en el promedio de edad de los 42 hombres (68,5 años) y de las 73 mujeres (68,9 años). El CEA fluctuó entre 0,2 y $271 \mathrm{ng} / \mathrm{ml}$ y el $43 \%$ tenía un valor superior a $5 \mathrm{ng} / \mathrm{ml}$. El tamaño tumoral promedio fue $5,6 \mathrm{~cm}$ (extremos 1,7-13). El compromiso en profundidad del tumor fue T2 en 3 casos, T3 en 93 casos y T4 en 19. El total de ganglios examinados fue 1.872 , con un promedio de 16,2 ganglios por paciente (extremos 3-54). El 58\% de los pacientes tuvo un recuento de ganglios igual o superior a 12. El promedio de ganglios positivos fue 3,4 (extremos 1-34). El índice ganglionar promedio fue 0,237 (DE: 0,197; extremos 0,031-0,882) y la mediana fue 0,1666 . El $74 \%$ de los pacientes tenía 1 a 3 ganglios positivos (N1) y el $26 \% 4$ o más ganglios positivos (N2).

El 38\% de los pacientes no recibió quimioterapia debido a las malas condiciones generales de salud, edad avanzada o rechazo del paciente. El seguimiento promedio fue de 67 meses (extremos 5-216), durante el cual se comprueba una recidiva en 37 pacientes y 29 fallecen por la enfermedad, 9 de los cuales (31\%) supera los 36 meses de obser- vación. Ocho pacientes reoperados por una recidiva están vivos con un seguimiento de 17, 47, 56, 57, $123,139,145$ y 184 meses, respectivamente. En el análisis univariado se demostró diferencias estadísticamente significativas en la supervivencia entre los pacientes N1 y N2 (estadios IIIA-B versus estadio IIIC) y entre los pacientes con $\mathrm{IG}>$ de 0,31 respecto de los con IG menor a ese valor (Tabla 1). En esta serie no hubo asociación entre la sobrevida y el CEA preoperatorio, el tamaño tumoral y el grado de diferenciación tumoral. Al contrastar la supervivencia de acuerdo al IG en cada subgrupo de acuerdo al número de LN positivos, apreciamos que existe una fuerte asociación entre el IG y la sobrevida sólo en los pacientes con más de 4 ganglios afectados (N2) (Tabla 2).

En forma global, el número de LN examinados considerado como variable dicotómica $(\geq 12$ ganglios) no tuvo una asociación estadísticamente significativa con la sobrevida (HR 12; IC 95\% 0,60 $-2,7 ; \mathrm{p}=0,52)$. Sin embargo, al introducir como variable el número de LN positivos y el IG, se aprecia una clara asociación, especialmente en el grupo con un recuento mayor de 12 ganglios (Tabla 3 ). Así, un paciente con una cosecha de LN menor de 12 y un $\mathrm{IG}>0,31$ tiene un riesgo de morir por $\mathrm{CC}$ 12,88 mayor respecto de uno con un $\mathrm{IG}<0,31$ con un recuento similar de ganglios. Por otra parte, los pacientes con un recuento menor de $12 \mathrm{LN}$ y más de 4 ganglios afectados (N2) tiene 7,2 veces más riesgo de morir por $\mathrm{CC}$ que un paciente $\mathrm{N} 1$ con el mismo recuento de LN.

El análisis de las curvas ROC mostró que el número de LN positivos y el IG son factores pronósticos relevantes. El área bajo la curva ROC del número de LN afectados (0,703; IC 95\%: 0,58-082) fue levemente mayor que el área bajo la curva ROC del IG (0,690; IC 95\%: 0,57-0,81), aunque sin dife-

Tabla 1. Características clínico-patológicas de $\mathbf{1 1 5}$ pacientes estadio III. Análisis univariado

\begin{tabular}{|lccccc|}
\hline Variable & n pacientes & Fallecidos & HR & IC 95\% & p \\
\hline Edad $(\leq 65 />65$ años $)$ & $35 / 80$ & $9 / 20$ & 1,26 & $0,55-2,72$ & 0,61 \\
Género (mujer/hombre) & $73 / 42$ & $21 / 8$ & 0,77 & $0,34-1,75$ & 0,54 \\
\hline CEA $*(\leq 5 />5 \mathrm{ng} / \mathrm{ml})$ & $58 / 44$ & $12 / 11$ & 1,41 & $0,62-3,20$ & 0,62 \\
Linfonodos N1/N2 & $85 / 30$ & $14 / 15$ & 4,06 & $1,95-8,48$ & $<0,0001$ \\
\hline TNM IIIA / IIIB / IIIC & $3 / 82 / 30$ & $0 / 14 / 15$ & 4,06 & $1,95-8,48$ & $<0,0001$ \\
BD-MD / Indiferenciados & $98 / 17$ & $24 / 5$ & 1,06 & $0,40-2,80$ & 0,89 \\
Tamaño $(\leq 5 />5$ cm) & $51 / 64$ & $14 / 15$ & 0,63 & $0,30-1,31$ & 0,22 \\
IG $(\leq 0,18 />0,18)$ & $73 / 42$ & $11 / 18$ & 1,96 & $0,92-4,96$ & 0,07 \\
IG $(\leq 0,31 />0,31)$ & $67 / 48$ & $12 / 17$ & 4,06 & $1,95-8,48$ & $<0,0001$ \\
\hline
\end{tabular}

*CEA medido en 102 pacientes. IG: índice ganglionar. 
Tabla 2. Análisis de sobrevida según número de LN positivos e índice ganglionar

\begin{tabular}{|lcccccc|}
\hline IG & n & Fallecidos & $\begin{array}{c}\text { Sobrevida } \mathbf{5} \text { años } \\
\mathbf{\%}\end{array}$ & HR & $\mathbf{9 5 \%}$ IC & p \\
\hline $\mathrm{pN} 1=85$ & 75 & 12 & 86 & 1,047 & $0,23-4,68$ & 0,952 \\
$\mathrm{IG} \leq 0,31$ & 10 & 2 & 77 & & & \\
$\mathrm{IG}>0,31$ & 9 & 1 & 86 & 9,57 & $1,18-77,6$ & 0,034 \\
$\mathrm{pN} 2=30$ & & & & & \\
$\mathrm{IG} \leq 0,31$ & 21 & 14 & 36 & & \\
$\mathrm{IG}>0,31$ & & & & & & \\
\hline
\end{tabular}

Tabla 3. Análisis de sobrevida según número de LN examinados, número de LN positivos e IG

\begin{tabular}{|c|c|c|c|c|c|}
\hline n LN examinados & n pacientes & Fallecidos & HR & IC $95 \%$ & $\mathbf{p}$ \\
\hline & \multicolumn{5}{|c|}{ IG como variable continua $(\leq 0,31 />0,31)$} \\
\hline \multirow{3}{*}{$\begin{array}{l}<12 \\
\geq 12\end{array}$} & 48 & 11 & 12,88 & $0,96-172$ & 0,053 \\
\hline & 67 & 18 & 247,1 & $22,05-2.768$ & $<0,0001$ \\
\hline & \multicolumn{5}{|c|}{ IG como variable dicotómica $(\leq 0,31 />0,31)$} \\
\hline$<12$ & 48 & 11 & 3,37 & $0,98-11,5$ & 0,053 \\
\hline \multirow[t]{2}{*}{$\geq 12$} & 67 & 18 & 6,71 & $2,44-18,4$ & $<0,0001$ \\
\hline & \multicolumn{5}{|c|}{ Número de LN positivos (N2/N1) } \\
\hline$<12$ & 48 & 11 & 7,2 & $2,17-23,9$ & 0,001 \\
\hline$\geq 12$ & 67 & 18 & 2,91 & $1,14-7,41$ & 0,025 \\
\hline
\end{tabular}

$\mathrm{IG}=$ índice ganglionar.

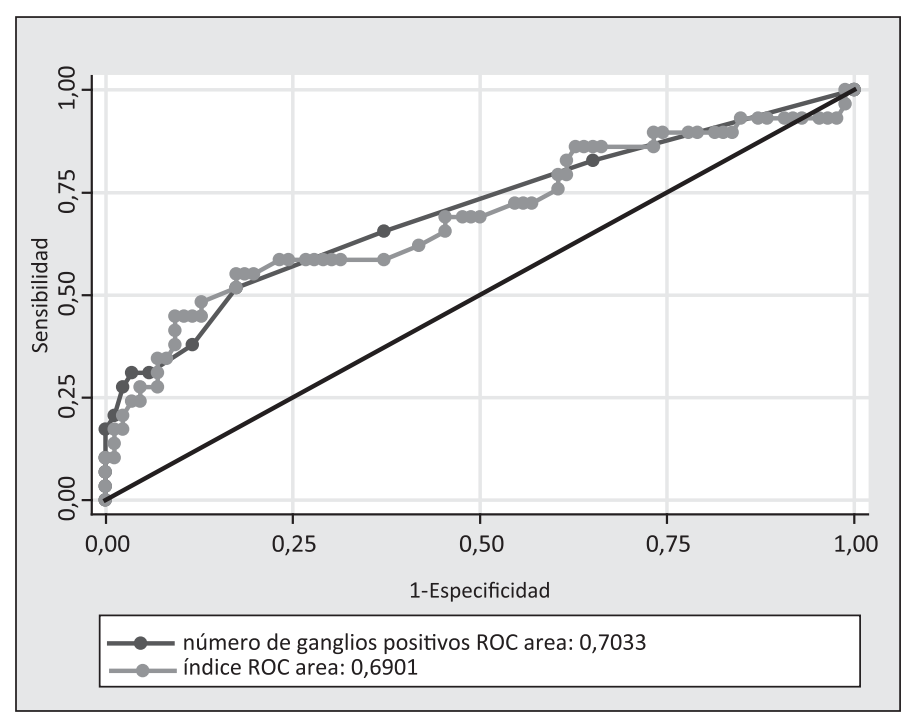

Figura 1. Curvas ROC: número LN positivos e IG. rencias estadísticamente significativas $(\mathrm{p}=0,63)$, lo que se aprecia en la Figura 1. En el análisis multivariado el número de LN metastásicos y el IG se mantienen como las únicas variables pronósticas relevantes. Al compararlas en forma dicotómica, el IG (OR: 19,96; IC 95\%: 1,51-253,6) muestra una mayor capacidad de discriminación que el número de LN afectados (OR: 2,55; IC 95\%: 0,86-7,55). Esta diferencia se acentúa cuando el recuento de LN supera los 12 ganglios examinados (Tabla 3). Las curvas de sobrevida estimadas por el método de Kaplan-Meier muestran que los pacientes con un IG mayor de 0,31 tienen un peor pronóstico que aquellos con un IG menor de 0,31 sólo en pacientes N2 (más de 4 LN positivos) (Figuras 2 y 3 ). 


\section{ÍNDICE GANGLIONAR Y NÚMERO DE LINFONODOS METASTÁSICOS COMO FACTORES PRONÓSTICOS EN ...}

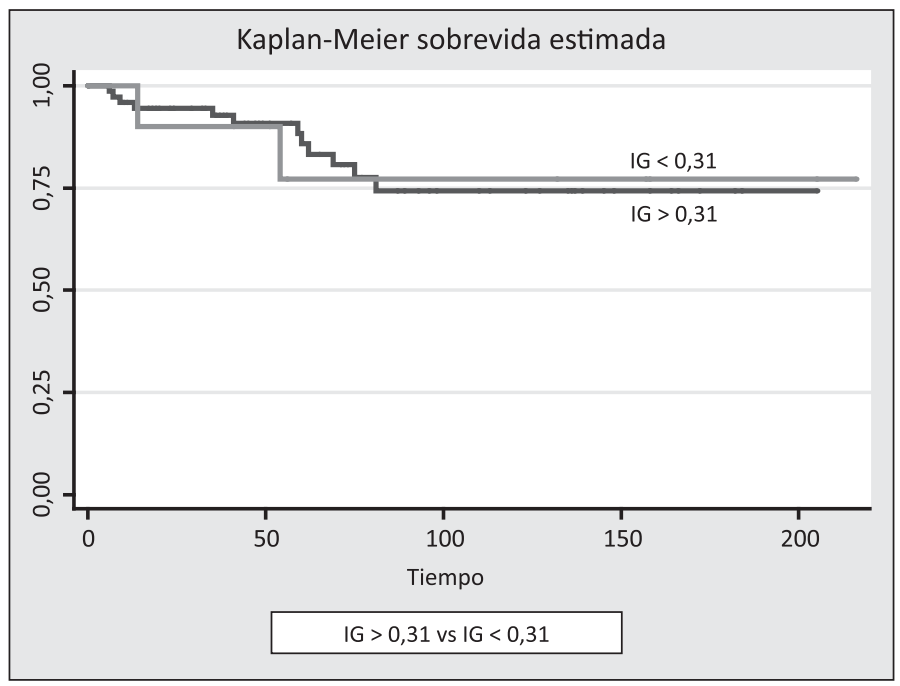

Figura 2. Sobrevida en pacientes N1 según IG.

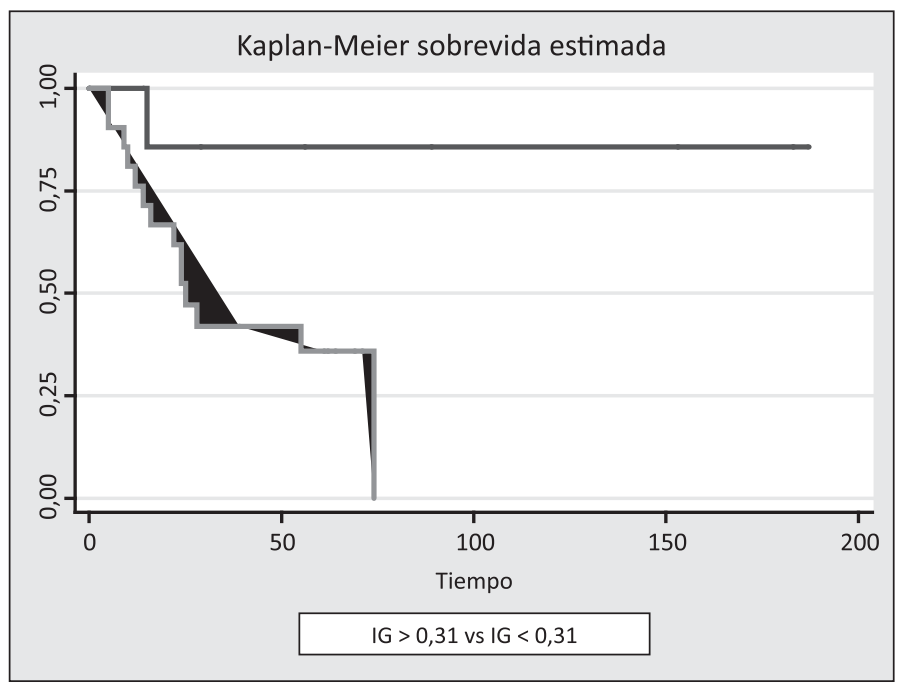

Figura 3. Sobrevida en pacientes N2 según IG.

\section{Discusión}

En esta serie se confirma el valor pronóstico relevante del compromiso ganglionar metastásico en los pacientes portadores de un CC intervenidos con intención curativa y los pacientes N2 tienen una menor sobrevida a largo plazo que los pacientes N1 en el análisis multivariado. Esta variable se ha estudiado intensamente, lo que ha conducido a la separación del estadio III en subgrupos con diferente pronóstico de acuerdo al número de LN afectados. En la sexta edición del TNM 2002, la AJCC distinguió entre los pacientes con 1 a 3 ganglios afectados (N1) de aquellos con 4 o más LN positivos (N2) ${ }^{3}$. La séptima edición del TNM $2010^{4}$ reagrupa los pacientes esta- dio III en 5 subgrupos, separando en el grupo N1 los pacientes con $1 \mathrm{LN}$ afectado (N1 a) de los pacientes con 2 ó 3 ganglios afectados (N1b). Además incluye en el grupo N1 a los pacientes T1/T2 con depósitos tumorales satélites en la subserosa sin LN metastásicos regionales, lo que denomina N1c. El grupo N2 lo divide en aquellos pacientes con 4 a 6 ganglios afectados (N2a) y los pacientes con 7 o más (N2b). La implicancia terapéutica de estos subgrupos es relevante, puesto que los esquemas adyuvantes en CC debieran adaptarse de acuerdo al pronóstico de cada paciente en particular según el compromiso de los LN, pero hasta ahora, no hay guías claras sobre cómo debiera modificarse dicha terapia. Los estudios poblacionales que han motivado estos cambios 
desgraciadamente no cumplen con los estándares mínimos para el tratamiento quirúrgico del CC. Así, el requisito de un número mínimo de 12 ganglios examinados, se cumplió sólo en el $37 \%$ de los casos en la serie SEER que analiza 116.995 pacientes con un promedio de $9 \mathrm{LN}$ por paciente ${ }^{19}$. Cifras similares encontraron en el estudio del National Cancer Database, USA ( $40 \%$ en 31.515 pacientes estadio II $)^{20}$, en el Registro de Cáncer de Ontario, Canadá $(27 \%$ de 1.789 casos estadio II $)^{21}$ y en el estudio poblacional francés $(19 \% \text { de } 851 \text { pacientes })^{22}$. En nuestra serie el promedio de ganglios examinados por paciente fue 16,2 y el $58 \%$ de la serie tuvo un recuento de 12 o más LN evaluados ${ }^{2}$, lo que es consistente con series provenientes de un centro asistencial específico $^{5-7,13,14}$.

Ello nos conduce a la segunda dimensión de esta variable que es el número de LN examinados como medida de la calidad de la cirugía del CC y una eventual asociación con una mejor sobrevida a largo plazo en pacientes estadio II y III ${ }^{1,5,6,18}$, lo que ha sido rebatido por otros autores ${ }^{23-25}$. En un estudio de 30.625 pacientes de la base de datos SEER, Wong ${ }^{23}$ concluye que el número de LN examinados no se asocia con el estadio ni la sobrevida a largo plazo. Wang $^{24}$, en un estudio de 24.477 pacientes estadio III de la misma base de datos señala el efecto confundente del IG y discute el recuento de LN como una medida de la calidad de la cirugía en cáncer de colon, similar a lo encontrado por Tsikitis ${ }^{25}$, en una serie clínica de 329 pacientes estadio III. Las causas de esta teórica mejor expectativa de vida a mayor cosecha de LN no están claras. Se ha sugerido que una resección más completa favorece una mejor estadificación (migración de estadios), un efecto estadístico y, menos probable, el efecto directo de reducir la carga tumoral del paciente. La asociación entre el recuento de LN y la sobrevida está afectado por otras variables relacionadas con el tumor (localización, inestabilidad microsatelital), el paciente (edad, obesidad, respuesta inmune) y del centro asistencial (volumen del hospital, experiencia del cirujano), lo que probablemente explique en parte las diferencias entre las series. Un análisis reciente de la base de datos del SEER, que analiza 131.953 pacientes, concluye que el número de $\mathrm{LN}$ evaluados ideal para una correcta estadificación con seguridad del $90 \%$ debe considerar el factor $\mathrm{T}$ y sugiere un recuento mínimo de $1,4,13$ y 21 ganglios para tumores T1, T2, T3 Y T4, respectivamente ${ }^{26}$.

El IG ha sido propuesto como una manera fácil de mejorar el valor predictivo del compromiso metastásico de los LN, lo que para algunos sería superior al número de LN positivos incorporado en el TNM ${ }^{11}$. El IG sería especialmente útil en aquellos pacientes con un recuento de LN menor de $12^{9}$, lo que no es efectivo en nuestra serie. De hecho, el IG mostró una mayor capacidad de discriminación cuando el recuento de LN fue igual o mayor de 12, lo que es altamente controversial en la literatura ${ }^{15}$. Existe una gran variabilidad en los estudios que incluyen pacientes con estadio II y IV, cáncer de recto, grandes series heterogéneas y diferentes valores del cutoff en la estratificación por cuartiles, lo que hace difícil la interpretación de los resultados ${ }^{13-15}$. Vaccaro ${ }^{13}$, analiza una serie de 362 pacientes estadio III del Hospital Italiano de Buenos Aires. Luego de reagrupar los pacientes en 2 grupos (IG mayor o menor de 0,25 ) concluye que el IG tiene un valor pronóstico independiente del número de LN examinados y del número de ganglios afectados. $\mathrm{Lee}^{27}$, estudia 201 pacientes estadio III confirmando el IG como un factor pronóstico de mayor relevancia que el número de LN metastásicos. Derwinger ${ }^{10}$, analiza 265 pacientes estadio III confirmando la mayor potencia pronóstica del IG respecto del número de LN positivos $(p<0,0002$ versus $p=0,04)$. Chin ${ }^{11}$, en un estudio de 624 pacientes no encuentra diferencias en la sobrevida entre pacientes N1 y N2 que tienen un $\mathrm{IG}<0,4$, concluyendo que el IG es un predictor más poderoso que el número de LN afectados, especialmente en los estadios IIIC (T3/T4-N2), similar a lo encontrado en nuestra serie. Park ${ }^{12}$, en una serie de 318 pacientes estadio III, llega a conclusiones similares sólo cuando el número de LN examinados es igual o mayor de 12 . Wang 9 , en el análisis de 24.477 pacientes estadio III del registro SEER, muestra diferencias significativas en pacientes estadios IIIB y IIIC según el cuartil del IG ( $p<0,0001)$, lo que es independiente del número de LN examinados $\mathrm{y}$ propone incorporar el IG al TNM.

La exacta relación entre el número de LN examinados, el número de LN positivos y el IG es difícil de precisar. Para algunos autores, el efecto en la sobrevida observado del número de LN examinados se debe al efecto confundente del IG, que parece ser un indicador pronóstico relevante ${ }^{24,25}$. Wang ${ }^{24}$, en el estudio señalado pone en duda la importancia del recuento ganglionar como variable pronóstica, lo que ha sido apoyado por los resultados de otras series clínicas $^{25,28}$, los cuales, a diferencia de los estudios de población, se consideran más controlados desde el punto de vista de la calidad de la cirugía y del análisis histopatológico. Las áreas bajo las curvas ROC en nuestro estudio no permiten sacar una conclusión definitiva al respecto y sugieren que existe una fuerte interacción entre las tres variables, con diferencias que no alcanzan significación estadística.

En nuestro estudio, el área bajo las curvas ROC, no demuestra una diferencia estadísticamente significativa entre el número de $\mathrm{LN}$ positivos y el IG como ocurre en la serie de Galicia, que analiza 145 
pacientes estadio III mediante el modelo de las curvas ROC y la separación de los pacientes en cuatro cuartiles ${ }^{14}$. Estas diferencias no tienen una explicación plausible. Aunque el cutoff utilizado en la serie italiana fue de 0,18 , este nivel en nuestra serie marca una tendencia pero no alcanza niveles estadísticamente significativos. Estos autores concluyen que el IG es un factor pronóstico independiente de la sobrevida libre de enfermedad, es más robusto que el número de LN positivos y es independiente del número de LN examinados.

En conclusión, en esta serie se manifiesta la relevancia pronóstica del compromiso linfonodal en pacientes con cáncer de colon estadio III. En nuestra serie, el IG es un factor pronóstico relevante en pacientes N2 (con 4 o más LN positivos), especialmente si el recuento de LN es igual o mayor de 12 , similar a otros estudios ${ }^{12}$. La fuerte interacción entre el número de LN examinados, el número de LN metastásicos y el IG, no permite concluir en forma categórica cual de ellos es el factor pronóstico más destacado. La quimioterapia adyuvante debiera ajustarse especialmente en los pacientes con un IG mayor de 0,31 y/o N2.

\section{Referencias}

1. Chang GJ, Rodríguez-Bigas MA, Skibber JM, Moyer VA. Lymph nodes evaluation and survival after curative resection of colon cancer: systematic review. J Natl Cancer Inst. 2007;99:666-72.

2. Bannura G, Cumsille MA, Barrera A, Contreras J, Melo C, Soto D. Resultados del tratamiento quirúrgico del cáncer de colon. Análisis de 439 pacientes. Rev Chil Cir. 2010;62:491-6.

3. Greene FL, Stewart AK, Norton HJ. A new TNM staging strategy for node-positive (stage III) colon canceran analysis of 50.042 patients. Ann Surg. 2002;236:41621.

4. Edge SB, Byrd DR, Compton CC, Fritz AG, Greene FL, Trotti A. (Eds). AJCC Cancer Staging Manual 2010. Springer;143-64.

5. Johnson P, Porter G, Ricciardi R, Baxter NN. Increasing negative lymph nodes count is independently associated with improved long term survival in stage IIIB and III C colon cancer. J Clin Oncol. 2006; 202:3570-5.

6. Le Voyer TE, Sigurdson ER, Hanlon AL, Mayer RJ, MacDonald JS, Catalano PJ, et al. Colon cancer survival is associated with increasing number of lymph nodes analyzed: a secondary analysis of intergroup trial INT0089. J Clin Oncol. 2003;21:2912-9.

7. Berger AC, Sigurdson ER, Le Voyer T, Hanlon A, Mayer RJ, MacDonald JS, et al. Colon cancer survival is associated with decreasing ratio of metastatic to examined lymph nodes. J Clin Oncol. 2005;34:8706-12.
8. Mammen JMV, James LE, Molloy M, Williams A, Wray CJ, Sussman JJ. The relationship of lymph node dissection and colon cancer survival in the Veterans Affairs Central Cancer Registry. Am J Surg. 2007;194:349-54.

9. Wang J, Hassett JM, Dayton MT, Kulayat MN. Lymph node ratio: role in the staging of node-positive colon cancer. Ann Surg Oncol. 2008;15:1600-8.

10. Derwinger K, Carlsson G, Gustavsson B. A study of lymph node ratio as a prognostic marker in colon cancer. Eur J Surg Oncol. 2008;34:771-5.

11. Chin C-C, Wang J-Y, Yeh C-Y, Kuo Y-H, Huang W-S, Yeh C-H. Metastatic lymph node ratio is a more precise predictor of prognosis than number of lymph node metastases in stage III colon cancer. Int J Colorectal Dis. 2009;24:1297-302.

12. Park IJ, Choi G-S, Jun SH. Nodal stage of stage III colon cancer: the impact of metastatic lymph node ratio. J Surg Oncol. 2009;100:240-3.

13. Vaccaro CA, Im V, Rossi GL, Ojea Quintana G, Benati M, Pérez de Arenaza D, et al. Lymph node ratio as prognosis factor for colon cancer treated by colorectal surgeons. Dis Colon Rectum 2009; 52:1244-50.

14. Galizia G, Orditura M, Ferraraccio F, Castellano P, Pinto $\mathrm{M}$, Zamboli A, et al. The lymph node ratio is a powerful prognostic factor of node-positive colon cancers undergoing potentially curative surgery. World J Surg. 2009;33:2704-13.

15. Ceelen W, Van Nieuwenhove Y, Pattyn P. Prognostic value of the lymph node ratio in stage III colorectal cancer: a systematic review. Ann Surg Oncol. 2010;17:2847-55.

16. Bannura G, Del Castillo C, Cumsille MA, Barrera A, Contreras J, Soto D, y col. Resultados de la quimioterapia adyuvante en cáncer de colon. Rev Chil Cir. 2008;60:403-9.

17. Harrell FE, Lee KL, Mark DB. Multivariable prognostic models: issues in developing models, evaluating assumptions and adequacy, and measuring and reducing errors. Stat Med. 1996;15:361-87.

18. Newson RB. Comparing the predictive power of survival models using Harrell's C or Somers' D. The Stata Journal 2010;10:339-58.

19. Baxter NN, Virnig DJ, Rothenberger DA, Morris AM, Jessurun J, Virnig BJ. Lymph node evaluation in colorectal cancer patients: a population-based study. J Natl Cancer Inst. 2005;97:219-25.

20. Swanson RS, Compton CC, Stewart AK, Bland KI. The prognosis of $\mathrm{T} 3 \mathrm{~N} 0$ colon cancer is dependent on the number of lymph nodes examined. Ann Surg Oncol. 2003;10:65-71.

21. Wright FC, Law CH, Last L, Khalifa M, Amaout A, Nasser $\mathrm{Z}$ et al. Lymph node retrieval and assessment in stage II colorectal cancer: a population-based study. Ann Surg Oncol. 2003;10:903-9.

22. Maurel J, Launoy G, Grosclaude P, Gignoux M, Arveux 
P, Mathieu-Daude H, et al. Lymph node harvest reporting in patients with carcinoma of large bowel: a French population-based study. Cancer 1998;82:1482-6.

23. Wong SL, Hong JI, Hollenbeck BK, Baser O, Birkmeyer JD. Hospital lymph node examination rates and survival after resection for colon cancer. JAMA 2007;298:2149-54.

24. Wang J, Kulaylat M, Rockette H, Hassett J, Rajput A, Dunn KB, Dayton M. Should total number of lymph nodes be used as a quality of care measure to stage III colon cancer? Ann Surg. 2009;249:559-63.

25. Tsikitis VL, Larson DL, Wolff BG, Kennedy G, Diehl N, Qin R, et al. Survival in stage III colon cancer is in- dependent of the total number of lymph nodes retrieved. J Am Coll Surg. 2009;208:42-7.

26. Gonen M, Schrag D, Weiser MR. Nodal staging score: a tool to assess adequate staging of node-negative colon cancer. J Clin Oncol. 2009;27:6166-71.

27. Lee H-Y, Choi H-J, Park K-J, Shin J-S, Kwon H-C, Roh $\mathrm{M}-\mathrm{S}$, et al. Prognostic significance of metastatic lymph node ratio in node-positive colon carcinoma. Ann Surg Oncol. 2007;14:1712-7.

28. Moug SJ, Saldahna JD, McGregor JR, Balsitis M, Diament RH. Positive lymph node retrieval ratio optimizes patient staging in colorectal cancer. $\mathrm{Br} \mathrm{J}$ Cancer 2009;100:1530-3. 